\title{
La politique scolaire rurale en Italie
}

\section{Marina Bertiglia et Novarino Panaro}

\section{OpenEdition}

\section{Journals}

Édition électronique

URL : http://journals.openedition.org/ries/3320

DOI : $10.4000 /$ ries.3320

ISSN : 2261-4265

\section{Éditeur}

Centre international d'études pédagogiques

\section{Édition imprimée}

Date de publication : 1 juin 1996

Pagination : 75-82

ISSN : 1254-4590

\section{Référence électronique}

Marina Bertiglia et Novarino Panaro, «La politique scolaire rurale en Italie », Revue internationale d'éducation de Sèvres [En ligne], 10 | 1996, mis en ligne le 30 juillet 2013, consulté le 14 novembre 2019. URL : http://journals.openedition.org/ries/3320

Ce document a été généré automatiquement le 14 novembre 2019.

() Tous droits réservés 


\title{
La politique scolaire rurale en Italie
}

\author{
Marina Bertiglia et Novarino Panaro
}

1 À partir de la loi Coppino (1880) et jusqu'au début des années soixante-dix, la politique scolaire italienne a cherché à étendre l'enseignement primaire aux plus petites communes. Dans toutes les agglomérations rurales et parfois même dans les hameaux les plus importants, des écoles primaires ont donc été progressivement implantées afin de permettre à tous les enfants d'être scolarisés jusqu'à la fin du cycle primaire, c'est-à-dire jusqu'à leur onzième année. La scolarisation obligatoire, qui avait été fixée par la même loi aux trois premières années du primaire, a été prolongée dans les années vingt à tout le cycle. De ce fait, l'administration scolaire et les pouvoirs locaux (la commune), conformément à leurs compétences respectives, ont eu à mettre en place un service scolaire dans les petites agglomérations rurales pour permettre à tous de satisfaire aux obligations définies par la loi.

2 La mise en œuvre d'un tel plan de développement s'est heurtée à de nombreuses difficultés d'ordre administratif et comptable.

3 Dans certains cas, particulièrement dans les petits villages de montagne, les enfants étaient trop peu nombreux pour que soient ouvertes des classes conformes aux normes définies au niveau national. Dans d'autres cas, les enfants recensés par le bureau de l'état civil étaient en nombre suffisant mais, en fait, peu d'entre eux s'inscrivaient à l'école, les parents préférant s'assurer l'aide de leur progéniture, fût-elle très jeune, aux travaux des champs ou aux soins des troupeaux.

Dans la plupart des cas, il s'agissait d'écoles situées dans des espaces ruraux qui n'étaient pas desservis quotidiennement par les transports publics et qui ne comptaient que quelques élèves par classe. Les critères retenus pour le fonctionnement des écoles situées dans de tels lieux étaient par conséquent bien différents de ceux qui avaient été définis au niveau national, et cela afin de maintenir les coûts du service scolaire (gratuit pour les familles) dans des limites compatibles. Ces critères ne concernaient pas seulement l'effectif minimum à partir duquel l'inspecteur d'académie compétent pouvait autoriser l'ouverture d'une classe ; ils concernaient aussi l'organisation de l'enseignement dans ces mêmes classes et par conséquent le nombre d'enseignants à affecter à chaque école. 
5 Au fil du temps, un modèle d'organisation spécifiquement adapté aux réalités rurales s'est imposé. Il prévoyait la constitution d'une unité d'enseignement au sein de laquelle un enseignant pouvait prendre en charge des élèves de niveaux différents. Bien que le travail d'un enseignant soit plus difficile dans une classe ainsi composée que dans une classe normale, aucun mauvais résultat n'a été enregistré, et cela en partie parce que l'effectif moyen de ces classes était réduit (10/15) en comparaison des effectifs habituels (25/30).

6 Les bons résultats obtenus, mais aussi l'exode massif des populations des campagnes vers les villes en pleine croissance industrielle, ont amené le législateur à s'occuper de la distribution du service scolaire en milieu rural dès 1957, année où fut promulguée une loi.

7 Ce texte réglementait le fonctionnement des écoles rurales en instituant le système des "pluriclasses ». Une "pluriclasse » peut être composée d'élèves de niveaux différents, d'enfants de première année associés à ceux de deuxième année par exemple, ou même, à la limite, à des enfants issus des quatre autres niveaux. Partout où il était possible de le faire, le regroupement des deux premiers niveaux (dit de " premier cycle») et celui des trois derniers (dit de "second cycle») ont été retenus. Une telle procédure visait à garantir au plus grand nombre la réelle jouissance du droit à l'instruction, ratifié par la Constitution italienne, ainsi qu'à favoriser le maintien des jeunes dans les zones rurales.

Ces prévisions normatives n'ont plus subi aucune modification jusqu'en 1990, année où, suite à la réforme de l'organisation de l'école primaire (loi n ${ }^{\circ} 148 / 90$ ), a été instauré un système d'enseignement modulaire, c'est-à-dire structuré sur un rapport de trois enseignants pour deux classes, ou de quatre enseignants pour trois classes.

Rappelons cependant que dans les années quatre-vingt, en Italie, un autre modèle d'organisation, appelé temps plein (TP), avait été introduit. Structurée sur un rapport de deux enseignants pour une classe, cette formule a d'abord été largement diffusée dans les grandes villes pour des raisons socio-économiques évidentes. Elle s'est ensuite étendue aux zones périphériques et, dans certains cas, même aux écoles qui fonctionnaient en pluriclasses. Dans ces dernières, le rapport enseignant/classes a donc été doublé indépendamment du nombre total d'élèves scolarisés.

On enregistre par conséquent aujourd'hui des situations très diverses :

- des «pluriclasses » uniques, avec un effectif variant de cinq à dix élèves, attribuées à un ou à deux enseignants (appelées, selon le cas, à « temps normal » et à « temps plein ») ;

- des « pluriclasses » avec un effectif variant de cinq à onze élèves, organisées en modules ;

- des « monoclasses » avec un effectif variant de cinq à vingt-cinq élèves (réduit à vingt en cas de présence d'un enfant porteur d'un handicap).

\section{L'exemple de Turin}

Dans la province de Turin, où les régions montagneuses occupent une partie importante du territoire et où l'on dénombre cent quatre petites agglomérations rurales pour un total de trois cent treize communes, le rapport global enseignant/élèves est celui qui figure au tableau suivant ${ }^{\circ}$ : 
Inspection académique de Turin

Classes et élèves pour l'année scolaire 1995/1996 (territoire métropolitain et province)

\begin{tabular}{|c|c|c|c|c|c|c|c|c|}
\hline & \multicolumn{2}{|c|}{ à T.N. ${ }^{1}$} & \multicolumn{2}{|c|}{ à T.P. ${ }^{2}$} & \multicolumn{2}{|c|}{ avec modules $^{3}$} & \multicolumn{2}{|c|}{ TOTAL } \\
\hline & Classes & Élèves & Classes & Élèves & Classes & Élèves & Classes & Élèves \\
\hline " Monoclasses " ........ & \multirow[t]{2}{*}{33} & 508 & \multirow[t]{2}{*}{2476} & 47320 & \multirow[t]{2}{*}{2140} & 32678 & \multirow[t]{2}{*}{4649} & 80506 \\
\hline Porteurs de handicaps ${ }^{4}$ & & 7 & & 906 & & 540 & & 1453 \\
\hline «Pluriclasses $" . . . . . . .$. & \multirow[t]{2}{*}{38} & 277 & \multirow[t]{2}{*}{23} & 212 & \multirow[t]{2}{*}{105} & 928 & \multirow[t]{2}{*}{166} & 1417 \\
\hline Porteurs de handicaps.. & & 2 & & 5 & & 11 & & 18 \\
\hline \multirow[t]{2}{*}{ Total général ........... } & \multirow[t]{2}{*}{71} & 785 & 2499 & 47532 & \multirow[t]{2}{*}{2245} & 33606 & \multirow[t]{2}{*}{4815} & 81923 \\
\hline & & 9 & & 911 & & 551 & & 1471 \\
\hline Élèves/classe & \multicolumn{2}{|c|}{$\begin{array}{c}\text { Monoclasses } \\
17,32\end{array}$} & \multicolumn{2}{|c|}{$\begin{array}{c}\text { Pluriclasses } \\
8,54\end{array}$} & \multicolumn{4}{|c|}{$\begin{array}{c}\text { Moyenne générale } \\
17,01\end{array}$} \\
\hline
\end{tabular}

1 T.N. signifie " temps normal " : P'emploi du temps prèvoit vingt-sept heures de cours hebdomadaires en premier cycle, trente en second cycle; tous les cours ont lieu le matin.

2 T.P. signifie « temps plein » : l'emploi du temps prévoit quarante heures hebdomadaires de cours traditionnels et d'activités complémentaires (travail dirigè, jeux d'éveil, etc.) ; les cours ont lieu le matin et l'après-midi.

3 Dans les classes avec modules, les différentes matières sont regroupées en cinq * domaines de disciplines " ; chaque enseignant ne s'occupe que d'un (parfois deux) domaines (par exemple mathèmatiques) et par conséquent intervient dans deux ou trois classes. L'organisation en modules prévoit donc deux enseignants pour trois classes, ou trois enseignants pour quatre classes.

4 La loi italienne prévoit que les élèves porteurs d'un handicap soient totalement intégrés dans les classes normales et qu'ils suivent les cours avec les autres enfants; si le handicap est lourd, un second enseignant, appelé enseignant de soutien, intervient dans la classe pour apporter une aide spécifique à l'enfant handicapé.

12 Une situation de ce genre entraîne évidemment un gaspillage de ressources très élevé. Depuis que chaque province a introduit, il y a quelques années, des taux moyens élèves/ classes et une limitation du nombre global de postes à attribuer, il est devenu indispensable d'équilibrer les intérêts des grandes et moyennes agglomérations avec ceux des zones plus périphériques.

Dans le cas de la province de Turin, pour l'année scolaire 1995-1996, l'indice susmentionné relatif à l'école primaire est de 17,4. L'effectif maximum d'enseignants prévu pour l'ensemble des besoins (classes expériences, laboratoires, soutien aux porteurs de handicaps, langues vivantes, cours pour adultes) est de 9320 unités pour un total de 81923 élèves. D'où la nécessité, pour qui dirige l'école, d'évaluer les priorités. Voici, à titre d'exemple, quelques données relatives aux besoins formulés par les chefs d'établissement :

- besoins en classes : 4819 ;

- besoins en postes d'enseignants : fonctionnement des modules classiques et des «temps plein » : 3506 ; soutien : 763 ; laboratoires : 56 ; langue vivante étrangère : 328 ; activités expérimentales : 31 ; cours pour adultes : 28 .

Ce tableau d'ensemble fait apparaitre une demande bien supérieure aux moyens disponibles. Par souci de transparence administrative, les besoins des petites et moyennes agglomérations sont satisfaits selon des paramètres précis. Outre le nombre d'élèves et les prévisions démographiques, sont pris en compte les conditions de transport, l'état des locaux, la distance à parcourir pour atteindre l'école la plus proche.

Pour l'instant, les chiffres signalent encore une diminution du nombre des classes traditionnelles et des pluriclasses, à un rythme plus rapide pour ces dernières : 


\begin{tabular}{|c|c|c|c|}
\hline Année scolaire & $1982-1983$ & $1990-1991$ & $1995-1996$ \\
\hline «Monoclasses » ...... & 7095 & 5008 & 4649 \\
«Pluriclasses » ....... & 380 & 213 & 166 \\
Total des classes ...... & 7475 & 5221 & 4815 \\
\hline
\end{tabular}
(vérifié chaque année) au-dessous duquel l'école ou la classe est supprimée. Ce souvent impossible l'élaboration d'un programme d'action sur plusieurs années et conduit parfois à la recherche laborieuse d'un nouvel enfant à inscrire, seul capable de garantir la survie de l'institution scolaire dans le territoire concerné.

Cette dernière remarque nous amène à nous pencher sur un phénomène de type socioculturel encore très répandu dans de nombreuses régions : la défense $\mathrm{du}$ « principe » de l'école dans la communauté. transportable »; ils ont besoin de grandir et de s'attacher au milieu où l'on veut les voir vivre et travailler.

(ans certaines vallées la situation soit maintenant stable, du moins en ce qui concerne la population scolaire du primaire, comme le montre le tableau suivant. difficultés d'extension du service scolaire aux petites agglomérations rurales ne concernent pas seulement l'enseignement primaire mais également les deux cycles du secondaire; le problème demeure particulièrement complexe pour les classes de collège comprises dans le cycle obligatoire.

Au début des années soixante-dix, en raison de l'allongement de l'obligation scolaire, fixée au terme de la troisième année du premier cycle - soit à quatorze ans - des classes de collège ont été ouvertes dans les agglomérations rurales d'au moins mille habitants.

Inspection académique de Turin Effectifs d'élèves

\begin{tabular}{|l|c|c|c|c|c|}
\hline \multicolumn{1}{|c|}{ Année scolaire } & 1991-1992 & $1992-1993$ & $1993-1994$ & $1994-1995$ & $1995-1996$ \\
\hline Communes de: & & & & & \\
\hline $\begin{array}{l}\text { Bobbio Pellice } \\
2 \text { «pluriclasses " } \\
1 \text { T.P. }\end{array}$ & 16 & 13 & 16 & 13 & 17 \\
\hline $\begin{array}{l}\text { Baussa di Torre Pellice } \\
4 \text { «pluriclasses " } \\
2 \text { T.P. }\end{array}$ & 34 & 37 & 37 & 37 & 36 \\
\hline $\begin{array}{l}\text { Angrogna } \\
2 \text { «pluriclasses " } \\
1 \text { T.P. }\end{array}$ & 20 & 22 & 25 & 26 & 19 \\
\hline
\end{tabular}



dispositions prévues pour la sauvegarde des territoires de montagne. Les exigences du service scolaire au niveau des classes de collège ne peuvent toutefois pas permettre (du moins pour l'instant) d'étendre à tout le cycle obligatoire la solution des "pluriclasses " adoptée dans le primaire, et cela même si ces deux segments du parcours scolaire sont 
désormais considérés comme un continuum d'opportunités éducatives en prise directe avec les réalités culturelles, régionales et sociales.

C'est dans cette logique que s'inscrivent les nouvelles mesures du gouvernement pour poursuivre cette politique. En effet, la loi du 31 janvier 1994, intitulée "Nouvelles dispositions pour les territoires de montagne ", déclare expressément qu'aux termes de l'article 44 de la Constitution italienne, « la sauvegarde et la revalorisation des territoires de montagne revêtent un intérêt prééminent pour la nation ».

L'article 21 prévoit explicitement que dans les agglomérations de montagne de moins de cinq mille habitants puissent être constitués des groupes scolaires comprenant une école maternelle, une école primaire et le premier cycle du secondaire auxquels soit affecté un directeur, conformément aux critères définis par le ministre de l'Éducation nationale. Il s'agit d'écoles administrativement autonomes et disposant d'organes de gestion en propre, tant didactiques que comptables, comme dans n'importe quelle autre école.

nembre 1994, le ministre de l'Éducation nationale a émis une ordonnance ministérielle spécifique par laquelle il fait état des procédures à suivre dans la constitution de telles écoles ainsi que dans la composition des nouvelles équipes pédagogiques à partir des équipes d'origine.

La nouvelle unité scolaire ainsi obtenue est couramment appelée "verticalisée " et parfois « intégrée ». L'ordonnance ministérielle prévoit deux cas de figure :

- le regroupement d'une école maternelle, d'une école primaire et du premier cycle du secondaire, aux termes de l'article 21 de la loi 97/1994;

- le regroupement d'une école maternelle, d'une école primaire et du premier cycle du secondaire, implanté de façon expérimentale dans une zone à risque sur le plan de la dispersion scolaire ou du comportement déviant.

La procédure prévoit, comme point de départ et condition sine qua non, l'entente préalable des écoles qui désirent se regrouper en unités « verticalisées ».

Cette condition première, liée au choix des conseils d'enseignants des écoles intéressées, n'est pas facile à obtenir car, la plupart du temps, la solution consistant à verticaliser différentes écoles n'est envisagée que lorsque ces dernières ont perdu leur autonomie et ont déjà été rattachées à d'autres écoles des communes voisines; leurs conseils d'enseignants se retrouvent donc amalgamés à celui de l'école d'accueil avec lequel ils forment un organe unique.

Si les différents maires des communes concernées arrivent assez facilement à concilier leurs intérêts, il n'en va pas de même pour les conseils d'enseignants appelés à décider. Dans ces derniers, dominent en nombre les enseignants de l'école qui a absorbé celle qui souhaite à présent être verticalisée avec d'autres. Le directeur et l'équipe de l'école d'accueil s'opposent souvent à la demande de verticalisation; en effet, en perdant les classes qu'elle a récupérées, l'école d'accueil risque elle-même de se retrouver rapidement avec un nombre de classes inférieur au minimum autorisé et, par conséquent, d'être rattachée à une autre école de la même commune.

Il est à souhaiter que de telles divergences d'intérêts, lorsqu'elles se manifestent, ne finissent pas par entraver le processus de verticalisation. Bien qu'il soit encore difficile, en l'état actuel des choses, d'harmoniser les différents conseils d'enseignants qui fusionnent en un seul organe décideur et de répondre de façon équilibrée aux attentes spécifiques de chaque cycle représenté, cette nouvelle organisation du parcours scolaire obligatoire est certainement mieux à même de traiter le problème de la formation des 
jeunes en milieu rural. Le passage d'un niveau d'études à un autre s'accompagne encore trop souvent aujourd'hui de difficultés dues essentiellement à la discontinuité des cycles.

La loi de finances de 1996 (art. 1) prévoit aussi un regroupement «à l'horizontale » dans le second cycle du secondaire pour des classes de filières différentes; puisse cette disposition être de bon augure.

Pour l'instant, dans le Piémont, on dénombre cinq écoles verticalisées, toutes situées dans les territoires de montagne de la province de Novara, dans la partie devenue autonome appelée province Cusioverbano-Ossola.

\begin{tabular}{|c|c|c|c|c|c|}
\hline École verticalisée de: & BACENO & ORNAVASSO & PIEDIMULERA & VANZONE & VAGOGNA \\
\hline Classes/sections & & & & & \\
\hline École maternelle ......... & 4 & 2 & - & 1 & 4 \\
\hline École primaire ........... & 15 & 14 & 20 & 14 & 11 \\
\hline $1^{\mathrm{er}}$ cycle du secondaire... & 6 & 9 & 8 & 4 & 6 \\
\hline École maternelle privée ... & 2 & 1 & 3 & 3 & - \\
\hline \multirow{4}{*}{$\begin{array}{l}\text { Enseignants secteur public } \\
\text { École maternelle } . . . \ldots \ldots \\
\text { École primaire ............. } \\
1^{\text {er }} \text { cycle du secondaire ..... }\end{array}$} & & & & & \\
\hline & 6 & 5 & - & 2 & 9 \\
\hline & 25 & 23 & 32 & 20 & 17 \\
\hline & 8 & 30 & 27 & 11 & 16 \\
\hline \multirow{5}{*}{$\begin{array}{c}\text { Élèves } \\
\text { École maternelle } \ldots . \ldots \ldots \\
\text { École primaire } \ldots \ldots \ldots \ldots \\
1^{\text {er }} \text { cycle du secondaire } \ldots \ldots \\
\text { École privée } \ldots \ldots \ldots \ldots \ldots\end{array}$} & & & & & \\
\hline & 70 & 38 & - & 17 & 74 \\
\hline & 146 & 170 & 200 & 103 & 107 \\
\hline & 114 & 154 & 94 & 82 & 103 \\
\hline & & & & & \\
\hline Communes concernées & 4 & 3 & 3 & 5 & 4 \\
\hline \multicolumn{6}{|l|}{$\begin{array}{c}\text { Nombre global } \\
\text { des naissances dans les } \\
\text { communes concernées }\end{array}$} \\
\hline $1993 \ldots \ldots \ldots \ldots \ldots \ldots$ & 29 & 50 & 44 & 17 & 37 \\
\hline $1994 \ldots \ldots \ldots \ldots \ldots \ldots$ & 33 & 42 & 30 & 31 & 46 \\
\hline $1995 \ldots \ldots \ldots \ldots \ldots \ldots$ & 25 & 33 & 41 & 25 & 32 \\
\hline
\end{tabular}

Les «écoles verticalisées » fournissent une réponse très satisfaisante, quoiqu'insuffisamment éprouvée, au problème de la survie de l'unité scolaire autonome en milieu rural. Leur organisation ne résout pas le problème du faible effectif d'élèves par classe, pour lequel les pluriclasses semblent être plus adaptées.

Les deux formules peuvent coexister et peuvent certainement, ensemble, apporter une réponse positive aux multiples raisons de maintenir une école dans les petites agglomérations rurales. Parmi tous les instruments de sauvegarde des espaces ruraux, la présence de l'école revêt une importance stratégique.

\section{RÉSUMÉS}

Au Piémont, la politique de service scolaire en zone de montagne impose la constitution d'unités d'enseignement regroupées aux plans administratif et pédagogique, avec intégration des moyens. 
Mais au-delà du simple calcul de coûts de l'application stricte des normes d'encadrement, les communautés locales défendent un attachement au milieu, un symbole de vie culturelle et leur propre développement.

INDEX

Index géographique : Italie, Piémont

Mots-clés : développement rural, école rurale, milieu rural

\section{AUTEURS}

MARINA BERTIGLIA

Inspecteur d'académie, Turin, Italie.

\section{NOVARINO PANARO}

Inspecteur, président de l'Istituto Regionale di Ricerca Sperimentazione Agiornamento Educativi (IRRSAE), Turin, Italie. 